\title{
Perfil clínico de pacientes com insuficiência cardíaca para embasar a prática clínica do enfermeiro
}

\author{
Luana Narjara Amaral*, Regimar Carla Machado**
}

${ }^{*}$ Enfermeira, Graduação em Enfermagem pela Universidade Federal do Rio Grande do Norte/UFRN, Natal/RN, ${ }^{*}$ Enfermeira, Pós-Doutorado em Cirurgia Translacional, Professora do Departamento de Enfermagem da Universidade Federal de São CarlosUFSCar e Professora do Programa de Pós-Graduação em Enfermagem da Universidade Federal do Rio Grande do Nortel UFRN, Natal/RN

\section{Resumo}

A enfermagem baseada em evidências fundamenta a prática clínica, sendo a revisão integrativa um de seus recursos. O presente estudo objetivou traçar o perfil clínico e sociodemográfico de pacientes com insuficiência cardíaca e discutir os resultados frente à prática clínica do enfermeiro. Trata-se de uma revisão integrativa da literatura realizada nas bases de dados da Cinahl, Pubmed e Lilacs, no período de junho de 2014 a fevereiro de 2015, e a amostra constituiu-se de 12 artigos. Os resultados das análises dos artigos demonstraram que a idade média de pacientes com insuficiência cardíaca era de 63 anos, predominância do sexo masculino, com cardiomiopatia isquêmica, classe funcional III e fator de risco hipertensão arterial sistêmica. Os sinais e sintomas de maior frequência foram a dispneia, a fadiga, o edema de membros inferiores, a dispneia paroxística noturna, estase jugular, ortopneia, tosse e precordialgia.

Palavras-chave: insuficiência cardíaca, sinais e sintomas, fatores de risco, cuidados de enfermagem, prática clínica baseada em evidências.

\section{Abstract \\ Clinical profile of patients with heart failure to support the clinical nurse's practice}

Evidence-based nursing substantiates the clinical practice and integrative literature review is one of its resources. This study aimed to determine the clinical and socio-demographic profile of patients with heart failure and discuss the results in clinical nursing practice. It is an integrative literature review conducted in the databases Cinahl, Pubmed and Lilacs, from June 2014 to February 2015, and the sample consisted of 12 articles. After analyzing the studies, the results showed that the average age of heart failure patients was 63 years old, predominantly male, with ischemic cardiomyopathy, functional class III and hypertension risk factor. The most frequent signs and symptoms were dyspnea, fatigue, lower limb edema, paroxysmal nocturnal dyspnea, jugular stasis, orthopnea, cough and chest pain.

Key-words: heart failure, signs and symptoms, risk factors, nursing care, evidence-based practice. 


\section{Resumen}

\section{Perfil clínico de pacientes con insuficiencia cardiaca para apoyar la práctica clínica del enfermero}

La enfermería basada en la evidencia fundamenta la práctica clínica y la revisión integradora es uno de sus recursos. Este estudio tuvo como objetivo conocer el perfil clínico y socio demográfico de pacientes con insuficiencia cardiaca y discutir los resultados en la práctica de clínica de enfermería. Se trata de una revisión integradora de la literatura realizada en las bases de datos Cinahl, Pubmed y Lilacs, de junio 2014 a febrero 2015, y la muestra consistió en 12 artículos. Después de analizar los artículos, los resultados mostraron que la edad media de los pacientes con insuficiencia cardíaca era de 63 ańos, predominantemente varones, con miocardiopatía isquémica, clase funcional III y factor de riesgo de hipertensión. Los signos y síntomas más frecuentes fueron disnea, fatiga, edema de miembros inferiores, disnea paroxística nocturna, estasis yugular, ortopnea, tos y dolor de pecho.

Palabras-clave: insuficiencia cardíaca, signos y síntomas, factores de riesgo, cuidado de enfermera, práctica basada en la evidencia.

\section{Introdução}

A Insuficiência Cardíaca (IC) caracteriza-se em uma síndrome clínica que evolui com alta morbidade e mortalidade [1]. Dados divulgados pelo Sistema Único de Saúde (SUS) demonstram alto índice de pacientes internados com diagnóstico de IC [2]. Nos Estados Unidos, cerca de 5,7 milhóes de pessoas convivem com a doença [3].

A doença manifesta-se mais evidentemente na população idosa, consistindo em um produto comum à maioria das doenças que acometem o sistema cardiovascular [4]. Em consequência da transição demográfica, na qual a média de idade da populaçáo está maior, as pessoas acima de 60 anos atingem atualmente $11 \%$ da populaçáo mundial, esse percentual deve passar para 20\% em 2050 [5]. Contudo, um aumento progressivo da população idosa leva a uma multiplicação do número de casos de IC.

A doença arterial coronariana, infarto do miocárdio, hipertensão arterial, valvopatias, cardiopatias congênitas, doença pulmonar grave e diabetes são condiçôes que podem promover um desgaste cardíaco. Assim o coração aumenta seu tamanho e frequência para suprir as necessidades do corpo, tornando a doença uma condição crônica [3].

A IC é uma síndrome sistêmica ocasionada por uma disfunção cardíaca, que promove um suprimento sanguíneo insuficiente, atendendo de forma ineficaz as necessidades metabólicas e tissulares do corpo $[1,3]$. Todavia, o débito cardíaco inadequado, e elevação da pressão pulmonar e sistêmica são as alteraçôes hemodinâmicas mais frequentes [1].

A isquemia é uma das causas mais comuns da IC descompensada na medida em que proporciona a redução da contratilidade miocárdica [3]. Nesse sentido, um estudo com pacientes que apresentavam IC descompensada e admitidos em uma unidade de emergência, demonstrou predomínio do sexo masculino e da etiologia isquêmica seguido de distúrbios valvares e chagásicos [4].

O exame clínico é essencial para estimar o grau de congestão na IC [6]. Sobretudo, a enfermagem é a classe que se mantém mais próximo desses pacientes, permitindo que o enfermeiro conheça suas alteraçóes e evolução, sejam elas físicas ou mentais [7]. Ressalta-se a importância dos membros da equipe de enfermagem estarem preparados para prestarem cuidados a esses pacientes, conhecendo as respostas do indivíduo frente à doença, assim como promover um atendimento de qualidade, que melhora a vida do paciente e amplie sua sobrevivência.

Diante do exposto, buscou-se por meio da revisão integrativa da literatura dados referentes à classe funcional, segundo os critérios da New York Heart Association (NYHA), as variáveis demográficas e os sinais e sintomas de pacientes com IC, permitindo, assim, analisar e sintetizar evidências para fundamentar a prática clínica de enfermagem.

O presente trabalho pretende traçar o perfil clínico e sociodemográfico de pacientes com insuficiência cardíaca e discutir os resultados do estudo frente à prática clínica do enfermeiro. 


\section{Métodologia}

Para a elaboração do estudo foram seguidas etapas que constituem o processo de elaboração de uma Revisão Integrativa [8-10]. (Figura 1)

Figura1 - Ełapas para elaboração de revisão integrativa da literatura. Natal/RN 2015.

Identificação do tema e elaboração da questão de pesquisa

A busca na literatura de forma exaustiva

Coleta de dados de cada artigo selecionado

Análise da qualidade metodológica dos estudos

Interpretação dos resultados

Síntese do conhecimento

O estudo foi orientado pela seguinte questão de pesquisa: Qual a produção científica sobre o perfil clínico e sociodemográfico de pacientes com insuficiência cardíaca para embasar a prática clínica do enfermeiro?

A pesquisa foi realizada no Cinahl (Cumulative Index to Nursing and Allied Health Literature), Pubmed (National Library of Medicine and Nattional Institutes of Health) e Lilacs (Literatura Latino-americana e do Caribe em Ciências da Saúde) no período de junho de 2014 a fevereiro de 2015.

Foram utilizados descritores controlados segundo Medical Subject Headings (MeSh): Heart Failure, Signs and Symptoms, Risk Factors; Nursing Care; Evidence-Based Practice, assim como pela classificaçáo dos Descritores em Ciências da Saúde (DeCS): Insuficiência Cardíaca; Sinais e Sintomas; Fatores de Risco; Cuidados de Enfermagem; Prática Clínica Baseada em Evidências. A pesquisa foi realizada a partir do cruzamento do descritor "insuficiência cardíaca" com os outros descritores, em língua portuguesa ou variaçóes em língua inglesa de acordo com o portal de pesquisa utilizado.

Como critérios de inclusão foram estabelecidos artigos científicos publicados de janeiro de 2009 até a data de coleta dos artigos disponíveis sobre forma de texto completo em línguas inglesa, portuguesa e espanhola, cujo objetivo continha como foco principal a caracterização de pacientes com IC quanto ao perfil clínico e sociodemográfico. Foram excluídas do estudo as produçóes duplicadas nas bases de dados e publicaçóes do tipo dissertação tese, editoriais, notas e opiniōes de especialistas.
Os artigos identificados pela estratégia de busca inicial foram avaliados independentemente por dois autores, mediante a leitura prévia dos títulos e resumos, e em casos de discordância entre os dois revisores com relação à inclusão de um trabalho, um terceiro revisor foi solicitado. Entretanto, selecionaram-se os estudos que atendiam aos critérios de inclusão, por meio de um instrumento validado [11] utilizado para revisão integrativa. Esse propôs questóes que permitiam o fornecimento de resultados válidos para pesquisa: título, periódico, autores, país, idioma, ano de publicação, objetivo, delineamento e caracterização da amostra quanto ao tipo de seleçáo, sexo, idade, principais manifestações clínicas e fatores de riscos associados.

As publicações foram classificadas em Nível de Evidencia (NE) segundo a Oxford Centre Evidence Based Medicine, "The Oxford Levels of Evidence2" que propóe distribuir de forma hierárquica a melhor evidência de acordo com o questionamento proposto. Esta forma de classificação proposta pela Oxford permite orientar a tomada de decisão final sem fazer recomendaçóes definitivas $[12,13]$.

A partir da leitura de cada artigo selecionado, foi possível caracterizá-los quanto aos autores, periódico e ano, objetivo e país de origem. Foram identificadas as características sociodemográficas, sinais e sintomas e, posteriormente, discutidos conforme o conteúdo disponível. Esta análise dos estudos possibilitou avaliar a evidência dos estudos subsidiando a prática clínica, facilitando a tomada de decisão do enfermeiro e a inquietação ao desenvolvimento de pesquisas futuras.

\section{Resultados}

Foram encontrados 146 artigos, destes o Pubmed contabilizou o maior número, um total de 125 , o Cinahl apresentou 15 e o Lilacs 6 publicaçóes, no entanto 134 foram excluídos em razão de não responderem ao questionamento que norteia esta pesquisa. $O$ restante foi lido na íntegra e, assim, restringido a seleção em 12 artigos, visto que atingiram os critérios de inclusão e preencheram corretamente o instrumento de coleta de dados aplicado para caracterizar as variáveis pertinentes a este estudo. $\mathrm{O}$ quadro 1 apresenta os estudos incluídos na pesquisa. 
Quadro 1 - Síntese dos estudos, considerando autores, periódico e ano, resultados e país de publicação. Natal/RN, 2015.

\begin{tabular}{|c|c|c|c|}
\hline Autores & Periódico e Ano & Resultados & País \\
\hline Mantovani et al. [14] & $\begin{array}{l}\text { Acta Paul Enferm, } \\
2015\end{array}$ & $\begin{array}{l}\text { Idade média de } 65 \pm 16 \text { anos, } 58 \% \text { do sexo masculino, } \\
50 \% \text { tinha até seis anos de estudo e ganhavam até três } \\
\text { salários mínimos. A etiologia mais prevalente da insu- } \\
\text { ficiência cardíaca foi a isquêmica, e a fração de ejeção } \\
\text { média da amostra foi de } 30 \pm 7 \% \text {, classe funcional II } \\
58,0 \% \text { A HAS e o DM estiveram presentes em } 62,5 \% \text { e } \\
37,5 \% \text {. }\end{array}$ & BRA \\
\hline Albuquerque et al. [15] & Arq Bras Cardiol, 2014 & $\begin{array}{l}\text { I Registro Brasileiro de Insuficiência Cardíaca - As- } \\
\text { pectos Clínicos, Qualidade Assistencial e Desfechos } \\
\text { Hospitalares: O total de } 1.263 \text { pacientes (64 } 16 \\
\text { anos, } 60 \% \text { mulheres) foi incluído a partir de } 51 \text { centros } \\
\text { de diferentes regiões do Brasil. As comorbidades mais } \\
\text { comuns foram HAS (70,8\%), DLP (36,7\%) e DM (34\%). } \\
\text { Em torno de 40,0\% dos pacientes apresentavam fun- } \\
\text { ção sistólica do ventrículo esquerdo normal. O estudo } \\
\text { BREATHE demonstrou a alta mortalidade intra-hospita- } \\
\text { lar dos pacientes admitidos com IC aguda no Brasil. }\end{array}$ & BRA \\
\hline Almeida et al. [16] & Esc Anna Nery, 2013 & $\begin{array}{l}\text { Idade média de } 60 \text { anos; } 59 \% \text { sexo masculino; renda } \\
1 \text {-2 salários mínimos; HAS 72,4\%; }\end{array}$ & BRA \\
\hline Shehab et al. [17] & $\begin{array}{l}\text { BMC Research Notes, } \\
2012\end{array}$ & $\begin{array}{l}\text { Idade média de } 57,8 \text { anos; } 79,8 \% \text { sexo masculino; } \\
\text { etiologia isquêmica } 44,6 \% \text {; classe funcional II } 62 \% \text {; } \\
\text { HAS } 64,3 \% \text {. }\end{array}$ & EUA \\
\hline Badin et al. [6] & $\begin{array}{l}\text { Rev Gaúcha Enferm, } \\
2011\end{array}$ & $\begin{array}{l}\text { Idade média } 63 \text { anos; } 61 \% \text { sexo masculino; renda } 1 \\
\text { salário mínimo 35,5\%; cor branca } 16 \% ; 5 \text { anos de } \\
\text { escolaridade; etiologia isquêmica } 34 \% \text {; classe funcio- } \\
\text { nal III } 67,5 \% \text {; manifestações clínicas: dispneia }(91,4 \%) ; \\
\text { edema }(63,7 \%) \text {; dispneia paroxística noturna (DPN) } \\
(87,5 \%) ; \text { fadiga }(67,3 \%) \text { estase jugular }(28,7 \%) \text {; ortop- } \\
\text { neia }(55,4 \%) .\end{array}$ & BRA \\
\hline Ambrosy et al. [18] & $\begin{array}{l}\text { American Journal of } \\
\text { Critical Care, } 2010\end{array}$ & $\begin{array}{l}\text { Idade média } 63,3 \text { anos; } 74,07 \% \text { sexo masculino; raça } \\
\text { caucasiana } 88,09 \% \text {; classe funcional IV 56,06\%; HAS } \\
74,41 \% \text {; manifestações clínicas: dispneia }(93,8 \%) \text {; ede- } \\
\text { ma (88,93\%;) estertores finos }(76,39) \text {; estase jugular } \\
(43,9 \%) .\end{array}$ & EUA \\
\hline Albert et al. [19] & $\begin{array}{l}\text { American Journal of } \\
\text { Critical Care, } 2010\end{array}$ & $\begin{array}{l}\text { Idade média } 61,6 \text { anos; } 65,2 \% \text { sexo masculino; etnia } \\
\text { branca } 58,3 \% \text {; etiologia isquêmica } 44,6 \% \text {; classe } \\
\text { funcional III 55\%; DM 36,6\%; manifestações clínicas: } \\
\text { dispneia (100\%); edema }(45,7 \%) \text {; DPN }(23,6 \%) \text {; tosse } \\
(18,8 \%) ; \text { pulso irregular }(19,9 \%) \text {; fadiga }(43,1 \%) \text {; pulso } \\
\text { irregular (19,9\%). }\end{array}$ & EUA \\
\hline Goldberg et al. [20] & Clin Cardiol, 2010 & $\begin{array}{l}\text { Idade média } 76 \text { anos; } 60,3 \% \text { sexo feminino; raça } \\
\text { caucasiana } 95 \% \text {; manifestações clínicas: dispneia } \\
(93 \%) \text {; edema (70\%); tosse }(51 \%) \text {; precordialgia }(30 \%) ; \\
\text { ortopneia }(37 \%) .\end{array}$ & EUA \\
\hline
\end{tabular}




\begin{tabular}{|c|c|c|c|}
\hline Song et al. [21] & $\begin{array}{l}\text { J Cardiovasc Nurs, } \\
2010\end{array}$ & $\begin{array}{l}\text { Idade média } 62 \text { anos; } 60,3 \% \text { sexo masculino; etiolo- } \\
\text { gia isquêmica } 49,4 \% \text {; classe funcional II 38,2\%; HAS } \\
53,9 \% \text {; manifestações clínicas: dispneia }(84,3 \%) \text {; ede- } \\
\text { ma }(56,5 \%) \text {; DPN }(59,1 \%) \text {; fadiga }(89,8 \%) \text {; ortopneia } \\
(59,6 \%) \text {. }\end{array}$ & EUA \\
\hline Balieiro et al. [22] & Arq Bras Cardiol, 2009 & $\begin{array}{l}\text { Idade média } 61 \text { anos; } 51,2 \% \text { sexo masculino; } 53 \% \\
\text { afrobrasileiros; etiologia hipertensiva } 46 \% \text {; classe fun- } \\
\text { cional I 35\%; HAS 90,3\%. }\end{array}$ & BRA \\
\hline Smolderen et al. [23] & $\begin{array}{l}\text { NIH Public Access, } \\
2009\end{array}$ & $\begin{array}{l}\text { Idade média } 66,9 \text { anos; } 78,2 \% \text { sexo masculino; hiper- } \\
\text { colesterolemia } 64 \% \text {. }\end{array}$ & EUA \\
\hline Margoto et al. [24] & $\begin{array}{l}\text { Rev Esc Enferm USP, } \\
2009\end{array}$ & $\begin{array}{l}\text { Idade média 58,1 anos; } 54 \% \text { sexo masculino; classe } \\
\text { funcional IV 91,8\%; HAS 35\%; manifestações clínicas: } \\
\text { dispneia }(85,2 \%) \text {; edema }(80,3 \%) \text {; estertores finos } \\
(75,4 \%) ; \text { tosse }(34,4 \%) \text {; hepatomegalia }(55,7 \%) \text {; pre- } \\
\text { cordialgia }(13 \%) \text {; ascite }(42,6 \%) \text {; fadiga }(67,3) \text {; estase } \\
\text { jugular }(24,6 \%) \text {; tontura }(5 \%) \text {; terceira bulha }(4,9 \%) \text {; } \\
\text { palpitação }(8,3 \%) \text {; ortopneia }(55,4 \%) \text {. }\end{array}$ & BRA \\
\hline
\end{tabular}

Quadro 2 - Nível de evidência conforme a Oxford Levels of Evidence 2, de acordo com o delineamento, amostra e forma de seleção. Natal/RN, 2015.

\begin{tabular}{|l|l|l|l|}
\hline \multicolumn{1}{|c|}{ Artigos } & \multicolumn{1}{|c|}{ Delineamento } & \multicolumn{1}{|c|}{ NE } \\
\hline$(14)$ & Estudo transversal Seleção & N3 \\
\hline$(14)$ & $\begin{array}{l}\text { Estudo observacional tipo registro, com } \\
\text { seguimento longitudinal }\end{array}$ & 32 pacientes/ aleatória & N3 \\
\hline$(14)$ & Estudo transversal & 37 pacientes/ conveniência & N3 \\
\hline$(15)$ & Estudo prospectivo & 353 pacientes / conveniência & N3 \\
\hline$(6)$ & Estudo transversal & 303 pacientes/ conveniência & N3 \\
\hline$(16)$ & Duplo cego, randomizado, controlado & 297 pacientes/ randômica & N1 \\
\hline$(17)$ & Descritivo transversal & 276 pacientes/ conveniência & N3 \\
\hline$(18)$ & $\begin{array}{l}\text { Estudo transversal e documental retros- } \\
\text { pectivo }\end{array}$ & 4537 pacientes/ conveniência & N3 \\
\hline$(19)$ & Estudo prospectivo & 421 pacientes/ conveniência & N3 \\
\hline$(20)$ & Estudo transversal & 166 pacientes/ conveniência & N3 \\
\hline$(21)$ & Observacional Transversal & 188 pacientes/ aleatória & N1 \\
\hline$(22)$ & Descritivo exploratório & 61 pacientes/ conveniência & N3 \\
\hline
\end{tabular}

Quanto aos objetivos discutidos nas pesquisas levantadas, dos artigos selecionados, $60 \%$ focaram as manifestaçóes clínicas dos pacientes e $40 \%$ dos estudos focaram os fatores de risco como principal objetivo.

Constatou-se no quadro 2 que a maioria dos artigos, referente a essa temática, apresenta níveis de evidência intermediário segundo a Oxford Levels of Evidence 2 [10].

A tabela I mostra a média de idade referente aos artigos encontrados. Estes atingiram percentis de 57 a 66 anos. Pode-se perceber, também, que os mais atingidos com a doença são os homens, representando mais da metade da população total de doentes. As variáveis, raça, renda, escolaridade, etiologia, classe funcional e fatores de risco, foram referidos de forma irregular nos estudos, sendo, portanto, apresentada apenas as que mais se destacaram.

Tabela I - Características sociodemográficas e clínicas abordadas nos estudos selecionados. Natal/RN, 2015.

\begin{tabular}{ll}
\hline \multicolumn{1}{c}{ Variáveis } & \multicolumn{1}{c}{ Características } \\
\hline Idade média & 62,84 anos* \\
Sexo masculino & $62,2 \% *$ \\
Raça & Caucasiana \\
Renda & $1-2$ salários mínimo... \\
Escolaridade & Primeiro grau incompleto... \\
Etiologia & Isquêmica... \\
Classe funcional & III... \\
Fatores de Risco & HAS... \\
\hline
\end{tabular}

*Média referente ao número de publicações do estudo; ...

Variável destaque em sua maioria nos estudos revisados. 
Tabela II - Distribuição dos sinais e sintomas de maior evidência nos estudos. Natal/RN, 2015.

\begin{tabular}{lc}
\hline \multicolumn{1}{c}{ Sinais e Sintomas (\%) } & Artigos revisados \\
\hline Dispneia $(91,3 \%)$ & $(6),(16),(17),(18),(19)$, \\
Fadiga $(66.8 \%)$ & $(6),(17),(19),(22)$ \\
Edema $(66,7 \%)$ & $(6),(16),(17),(18),(19)$, \\
DPN*(56,7\%) & $(22)$ \\
Estase jugular $(53,3 \%)$ & $(6),(17),(19)$, \\
Ortopneia(49,3\%) & $(6),(22)$ \\
Tosse $(34,7 \%)$ & $(17),(18),(19),(22)$ \\
Precordialgia $(21,5 \%)$ & $(17),(18),(22)$ \\
\hline
\end{tabular}

*Dispneia paroxística noturna.

Verificou-se na tabela II, uma grande frequência de sintomas respiratórios dos pacientes com Insuficiência Cardíaca, sendo estes os de maior significância, dispneia, por exemplo, aparece com $91,3 \%$ de frequência.

\section{Discussão}

A Insuficiência Cardíaca (IC) é uma síndrome que compromete particularmente à qualidade de vida, reconhecida como um problema de relevância em saúde pública em todo o mundo. Um conjunto de fatores que contribuem para a ocorrência dessa síndrome desafia as equipes de saúde. O maior desafio consiste no tratamento e nos cuidados mais adequados aos pacientes, para manter a estabilidade clínica, reduzir o tempo de internação e proporcionar melhor qualidade de vida.

A literatura investigada neste estudo demonstra características sociodemográficas e perfil clínico de pacientes que apresentam insuficiência cardíaca. Sobretudo, enfatiza a saúde baseada em evidências, que propóe o uso consciente da melhor e mais recente resposta na literatura científica para a tomada de decisáo sobre o cuidar individual do paciente [10].

Os estudos avaliados demonstraram que a média de idade atingiu 62,2 anos, nos pacientes acometidos com IC, caracterizando uma populaçáo idosa [6,16,18-23]. Prevaleceu nos achados, a raça caucasiana [18,20], e renda equivalente de 1-2 salários mínimos. O primeiro grau incompleto foi destaque quanto ao nível de escolaridade, realçando a dificuldade de acesso à educação. Estudo realizado em 2008 [25] demonstra que a principal intervenção de enfermagem foi de caráter educativo, com capacitação do desenvolvimento do autocuidado. A menor escolaridade do paciente requer uma maior atenção acerca das instruçóes dadas sobre a doença e formas como manter uma melhor qualidade de vida.

Outro fator importante destacado na pesquisa é a prevalência do sexo masculino, o dado corrobora o divulgado pelo SUS, o qual mostra uma pequena ascensão em relação ao número de mulheres que se internam com a doença [2]. Outrossim, pode estar relacionado ao fato de que o homem náo priorize os cuidados com a própria saúde. Cabe ao profissional conhecer as proposiçôes acerca da saúde cardiológica do programa de saúde do homem, já que propóe uma melhor forma de atender e prevenir a doença nessa população [26].

Dentre as possíveis etiologias da IC, mais da metade dos estudos apresentaram etiologia isquêmica como a principal causa da doença $[6,7,14,19,21]$. Definir a etiologia é essencial na observação dos pacientes com IC, ajuda na avaliação da doença e direciona o tratamento. Angina de peito, antecedente de infarto do miocárdio, fatores de risco para aterosclerose, área inativa em eletrocardiograma e presença de disfunção segmentar ao ecocardiograma são sinais sugestivos de etiologia isquêmica [1].

Os estudos relacionaram a hipertensão arterial sistêmica (HAS), dislipidemia, diabetes mellitus (DM) e tabagismo como principais fatores de risco da insuficiência cardíaca, dos quais a HAS o mais importante deles [16-18,21-22,24]. A hipertensão é um grande problema de saúde pública no mundo, apresentando alta morbimortalidade. A pressão sistólica (PS) acima de $115 \mathrm{mmHg}$ e a pressão diastólica (PD) de $75 \mathrm{mmHg}$ aumentam constantemente o risco de eventos cardiovasculares [27]. A redução dos índices pressóricos, por meio de tratamento farmacológico e não farmacológico, é a melhor forma de prevenir o aparecimento da doença.

Ao longo do tempo, a HAS não controlada pode causar disfunção no ventrículo esquerdo ajudando o desenvolvimento da cardiopatia hipertensiva, portanto um dos fatores etiológicos da insuficiência cardíaca. Além disso, é considerada fator de risco para doenças decorrentes de aterosclerose e trombose, como a isquemia miocárdica, principal etiologia da IC [27].

Salienta-se a importância do profissional de saúde conhecer a hipertensão arterial sistêmica como principal fator de risco para a insuficiência cardíaca e a cardiomiopatia dilatada, permitindo, assim, buscar intervençóes na causa primária da doença, conduzir uma melhor terapêutica e dar orientação aos pacientes que apresentam pré-disposição a doença. 
$\mathrm{Na}$ avaliação da classe funcional, foi visto com maior frequência a classe funcional III $[6,19]$, em que os sinais e sintomas apresentam em pequenos esforços. Segundo a New York Heart Association (NYHA), os indivíduos com IC são classificados em quatro classes, permitindo avaliar o efeito dos sintomas da doença cardíaca, e subdividindo o grau de limitação imposto por ela para atividades cotidianas [1]. Essa classificação concede ao enfermeiro capacidade para avaliar a resposta diagnóstica do paciente, frente ao tratamento realizado, e intervir para que possa recuperar sua capacidade de realizar as atividades do cotidiano.

A maior presença da classe funcional III está associada à subutilização da terapia medicamentosa e náo medicamentosa dos pacientes com IC [2,2425]. A necessidade de manobras dinâmicas que melhor avaliem esses pacientes no momento da alta, a implementação de intervençôes que permitam melhor acompanhamento e controle dos pacientes com IC e uma consulta de enfermagem, com cunho educativo voltado para o autocuidado e adesão ao tratamento, interferem no número de internaçóes e reinternaçóes por complicação [24-25,28].

A partir da análise dos estudos, observou-se que os sinais e sintomas encontrados com maior prevalência foram dispneia, fadiga, edema, DPN e estase jugular [6,18-21,24]. Essas manifestaçóes promovem modificaçóes no padráo de vida desses pacientes, levando a uma incapacidade crescente para executar suas atividades cotidianas [29].

Os sinais e sintomas hemodinâmicos comprovam que a forma congesta da IC é a mais prevalente. Dispneia, fadiga, DPN, estase de jugular, ortopneia e tosse caracterizam o estado clínico de congestáo pulmonar. O desconforto respiratório causado reduz a tolerância à atividade, com os pulmóes congestos, o coração é incapaz de manter o débito cardíaco suficiente para satisfazer as necessidades teciduais reduzindo fluxo sanguíneo periférico [30].

Algumas intervençóes da equipe de enfermagem frente a essas queixas são necessárias, como examinar a condição pulmonar e de perfusão, observar oximetria de pulso $<90 \%$, avaliar o estado hemodinâmico e eletrolítico, manter leito elevado a $90^{\circ}$ e monitorar níveis de consciência, pressáo arterial, pulso, temperatura e padrão respiratório [30-31]. Esses cuidados permitem melhorar o prognóstico desses pacientes assim como manter um maior conforto respiratório. Assim, cabe ressaltar a importância da consulta periódica de enfermagem, avaliaçáo física minuciosa e acompanhamento da evolução da doença pelo enfermeiro.

O desenvolvimento do edema, por sua vez, está relacionado com a congestáo sistêmica. Com o volume de sangue diminuído, devido ao baixo suprimento do coraçáo, os rins passam a reter água e sódio acumulando-se no espaço intersticial [30]. Controlar ingestão hídrica, avaliar extensão e severidade do edema, investigar fatores desencadeantes e discutir terapêutica são formas de reduzir esta clínica do paciente [29-30].

A partir da identificação dos sinais e sintomas prevalentes na doença e do melhor conhecimento sobre a síndrome $[28,31]$, poderá favorecer um melhor entendimento das necessidades do paciente em sua internaçáo e fazer uma orientação planejada no momento da alta hospitalar de aspectos importantes, como, a alimentação, o repouso, a atividade física, os medicamentos, e demais cuidados relativos à doença, permitindo assim que o enfermeiro interfira nos riscos de reinternação e de mortalidade, promovendo uma melhor qualidade de vida.

A literatura identificou as principais características sociodemográficos e perfil clínico de pacientes com IC. Com base nestas informaçóes, possivelmente forneceu dados para estabelecer melhores cuidados a estes pacientes subsidiando a prática clínica do enfermeiro.

\section{Conclusão}

Percebeu-se que os sinais e sintomas de maior frequência em pacientes com IC foram a dispneia, a fadiga, o edema de membros inferiores, a dispneia paroxística noturna, a estase jugular, a ortopneia, a tosse e a precordialgia. Idade média de 63 anos, predominância do sexo masculino, com cardiomiopatia isquêmica, classe funcional III e fator de risco hipertensão arterial sistêmica.

Diante da prevalência das características encontradas, a evidência constatada se faz importante, já que permite ao enfermeiro identificar a doença previamente, bem como intervir rápido e corretamente ante as manifestaçóes da doença.

Este estudo limita-se na identificação das características enfocando a insuficiência cardíaca como doença única, ignorando as etiologias que levam a IC. A construção de um novo estudo voltado para cada doença, a fim de que se possam entender as necessidades de cada grupo e fornecer evidências mais definidas são sugestóes para novas pesquisas. 


\section{Referências}

1. Bocchi EA, Marcondes-Braga FG, Ayub-Ferreira SM, Rohde LE, Oliveira WA, Almeida DR, et. al. Sociedade Brasileira de Cardiologia. III Diretriz Brasileira de Insuficiência Cardíaca Crônica. Arq Bras Cardiol 2009;93(1):1-71.

2. Brasil. Ministério da Saúde. Datasus: informações de saúde. Brasília: Ministério da Saúde; 2014.

3. Bocchi EA, Marcondes-Braga FG, Bacal F, Ferraz AS, Albuquerque D, Rodrigues D et al. Sociedade Brasileira de Cardiologia. Atualização da Diretriz Brasileira de Insuficiência Cardíaca Crônica - 2012. Arq Bras Cardiol 2012;98(1):1-33.

4. Mangini S, Silveira FS, Silva CP, Grativvol PS, Seguro LFBC, Ferreira SMA et al. Insuficiência cardíaca descompensada na unidade de emergência de hospital especializado em cardiologia. Arq Bras Cardiol 2008;90(6):433-40.

5. Panel side event at 66th World Health Assembly. Functional decline and dependence in ageing populations. World Health Organization. Hague;2013

6. Badin AG, Linhares JCC, Linch GFC, Ruschel KB, Rabelo ER. Sinais e sintomas de pacientes com insuficiência cardíaca descompensada: inferência dos diagnósticos de enfermagem prioritários. Rev Gaúcha Enferm 2011;32(3):590-5.

7. Samanta M, Marques IR. Cardiomiopatia Hipertrófica: atualização e assistência de enfermagem. Rev Bras Enferm 2007;60(5):596-601.

8. Souza MT, Silva MD, Carvalho R. Revisão integrativa: o que é e como fazer. Einstein 2010;8(1):102-6.

9. Pompeo DA, Rossi LA, Galvão CM. Revisão integrativa: etapa inicial do processo de validação de diagnóstico de enfermagem. Acta Paul Enferm 2009;22(4):434-8.

10. Mendes KDS, Silveira RCCP, Galvão CM. Revisáo integrativa: método de pesquisa para a incorporação de evidências na saúde e na enfermagem. Texto Contexto Enferm 2008;17(4):758-64.

11. Ursi ES, Galvão CM. Prevençáo de lesóes de pele no perioperatório: Revisão integrativa da literatura. Rev Latinoam Enferm 2006;14(1):124-31.

12. Howick J, Chalmers I, Glasziou P, Greenhalgh T, Heneghan C, Liberati A et al. The 2011 Oxford CEBM Evidence Levels of Evidence (Introductory Document). Oxford Centre for Evidence-Based Medicine; 2011.

13. OCEBM Levels of Evidence Working Group. The Oxford Levels of Evidence 2. Oxford Centre for Evidence-Based Medicine; 2011.

14. Albuquerque DC, Souza Neto JD, Bacal F, Rohde LEP, Bernardez-Pereira S, Berwanger O et al. I Registro Brasileiro de Insuficiência Cardíaca - Aspectos Clínicos, Qualidade Assistencial e Desfechos Hospitalares. Arq Bras Cardiol 2015;104(6).

15. Mantovani VM, Ruschel KB, Souza EN, Mussi C, Rabelo-Silva ER. Adesão ao tratamento de pacientes com insuficiência cardíaca em acompanhamento domiciliar por enfermeiros. Acta Paul Enferm 2015;28(1):41-7.

16. Almeida GAS, Teixeira JBA, Barichello E, Barbosa MH. Perfil de saúde de pacientes acometidos por insuficiência cardíaca. Esc Anna Nery Rev Enferm 2013;17(2):328-35.
17. Shehab A, Dabbagh B, Almahmeed W, Bustani N, Nagelkerke N, Yusufali A et al. Characteristics and in-hospital outcomes of patients with acute coronary syndromes and heart failure in the United Arab Emirates. BMC Res Notes 2012;5(534):2-7.

18. Ambrosy AP, Pang PS, Khan S, Konstam MA, Fonarow GC, Traver Brian, et al. Clinical course and predictive value of congestion during hospitalization in patients admitted for worsening signs and symptoms of heart failure with reduced ejection fraction: findings from the Everest trial. Eur Heart J 2013;4:835-43.

19. Albert N, Trochelman K, Li J, Lin S. Signs and symptoms of heart failure: are you asking the right questions? Am J Crit Care 2010;19:443-52.

20. Goldberg RJ, Spencer FA, Szklo-Coxe M, Tisminetzky $M$, Yarzebski J, Lessard D,et al. Symptom presentation in patients hospitalized with acute heart failure. Clin Cardiol 2010;33(6):E73-80.

21. Song EK, Moser DK, Rayens MK, Lennie TA. Symptom clusters predict event-free survival in patients with heart failure. J Cardiovasc Nurs 2010;25(4):284-91.

22. Balieiro HM, Osugue RK, Rangel SP, Brandão R, Balieiro TL, Bernardez $S$, et al . Perfil clínico-demográfico e indicadores de qualidade da insuficiência cardíaca em uma área rural. Arq Bras Cardiol 2009;93(6):687-91.

23. Smolderen KG, Pelle AJ, Kupper N, Mols F, Denollet J. Impact of peripheral arterial disease on health status: A comparison with chronic heart failure. J Vasc Surg 2009;50(6):1391-8.

24. Margoto G, Colombo RCR, Gallani MCBJ. Características clínicas e psicossociais do paciente com insuficiência cardíaca que interna por descompensação clínica. Rev Esc Enferm USP 2009;43(1):44-53.

25. Bento VFR, Brofman PRS. Impacto da Consulta de enfermagem na frequência de internaçôes em pacientes com insuficiência cardíaca em Curitiba - Paraná. Arq Bras Cardiol 2009;92(6):490-6.

26. Chagas ACP, Zilli EC, Ferreira J FM, Moretti MA, Ramos RF. Saúde cardiovascular do homem brasileiro: visão da Sociedade Brasileira de Cardiologia. Arq Bras Cardiol 2009;93(6):584-7.

27. Santos ACS, Santo FHE, Pestana L, Daher DV, Santana R. Insuficiência cardíaca: estratégias usadas por idosos na busca por qualidade de vida. Rev Bras Enferm 2011;64(5):857-63.

28. Pereira JMV, Cavalcant ACD, Santana RF, Cassiano KM, Queluci GC, Guimarães TCF. Diagnósticos de enfermagem de pacientes hospitalizados com doenças cardiovasculares. Esc Anna Nery Rev Enferm 2011;15(4):737-45.

29. Araújo AA, Nóbrega MML, Garcia TR. Diagnósticos e intervençóes de enfermagem para pacientes portadores de insuficiência cardíaca congestiva utilizando a CIPE. Rev Esc Enferm USP 2013;47(2):385-92.

30. Azzolin K, Souza EN, Ruscel KB, Mussi CM, Lucena AF, Rabelo ER. Consenso de diagnósticos, resultados e intervençóes de enfermagem para pacientes com insuficiência cardíaca em domicílio. Rev Gaúcha Enferm 2012;33(4):56-63.

31. Machado CGD, Wansing GB, Klein C, Moraes MAP, Rabelo-Silva ER. Conhecimento do enfermeiro sobre insuficiência cardíaca em hospital geral. Rev Enferm UFSM 2014;4(4):710-7. 